Besides the very adoquate review of the current literature there are a number of special articles describing veterinary work in the United States of America, Dənmark, Sweden, Canada, Australia, New Zealand, Central and South Africa. That by Smithcors about the United States of America is an exeellent introductory article which sets a very high standard for those to follow. Various aspects of veterinary practice in Great Britain are also covered under the headings of country practice, horse practice and small-animal prastice. Here the contributors are too vague and in none does the standard approach that of Smithcors or of the introduction to greyhound racing by Bateman. In the next edition the editor might pay attention to some of the subjects discussed in his own preface. Contributions relating new methods of husbandry and feeding to the wide range of standards of management existing in Great Britain would certainly become a most fruitful new venture. It should be noted that the small list of new publications included in the book cannot be con. sidered by any means exhaustive.

This annual is strongly recommended to all those many veterinary surgeons and field research workers who lack adequate library facilities or a comprehensive abstract service.

G. N. Gould

\section{STEROID CHEMISTRY}

\section{Steroids}

By Prof. Louis F. Fieser and Mary Fieser. Pp. xvii+945. (New York: Reinhold Publishing Corporation; London: Chapman and Hall, Ltd., 1959.) 144s, net.

7 HIS book represents the tourth edition of one first published in 1936 under the title "Natural Products Related to Phenanthrene", the third edition of which was published in 1949. The present book deals entirely with the chemistry of the steroids, which have become by far the most important group of naturally oceurring phenanthrene derivatives.

The reviewer's task is a pleasant one because this is a superb presentation, both for the specialist and for the general reader. Biological and commercial interest in the steroids over the past ten years has stimulated a vast amount of chemical research. This might have proved dull, but in fact it has been intimately connected with new applications of physical techniques (for example, infra-red spectra and rotatory dispersion), new theoretical ideas such as conformational analysis, and the development of new reagents. It might fairly be claimed that the steroids have set the pace for the development of alicyclic chemistry over the past decade.

This book can be read with pleasure and profit by workers in any field of organic chemistry, including the specialist on reaction mechanisms, the synthetic organic chemist, the worker who is concerned with the application of new instrumental methods, etc. Not only does this book review most admirably the literature of the subject; but also the authors in many places add their own interpretations and generalizationsand furthermore they pose new problems for research.

All aspects of steroid chemistry are covered, and although the book does not pretend to be a complete catalogue of all the facts, the wealth of detail is such that it may be feared that many research students will be tempted not to bother to look at the original literature.
The general ehapters of the book cover the following topics: Chapters 1-2, introduction and structure of the steroid skeleton; 5, physical methods of characterization; 6 , oxidation; 7 , enes and ols; 8 , ketones; 9, displacements and rearrangements; 10 , stereochemical correlations and conventions.

The specialized chapters deal with all the main groups of steroids as follows: Chapter 4, vitamin D ; 11, sterols; 12, methylsterols (including the tetraeyclic triterpenoids) ; 13, biosynthesis of cholesterol ; 14, bile acids and alcohols; 15, cetrogens; 16 , androgens ; 17, progestogens ; 18, homo- and nor- steroids; 19 , adrenocortical hormones; 20 , cardiac-active principles; 21, sapogenins; 22 , alkaloids.

The book is lavishly illustrated with structural formulæ, many of them three-dimensional. A pleasant feature is the inclusion of many personal notes about workers in the field; such insertions would be annoying if they took the place of good factual and theoretical information, but when they are added to a fine scientific presentation, they contribute greatly to the interest of the book.

One of the most important facts about this book is that it is so up to date. The date of publication in the United States was July 1959 ; there are many 1959 references, and others to work then unpublished. Many monographs to-day suffer so much from serious delays in editing, printing, revision, etc., that this book must provide authors and publishers with a model of what can be achieved; typographical accuracy and the clarity of the formula leave nothing to be desired.

The more one reads of the book the more one is amazed at the way in which the two authors have combined a broad view of the subject with a wonderful grasp of detail. It is significant that in a book of 900 pages they have not followed the common trend of making it a collaborative effort. They have, however, obtained the help of colleagues in the field in another way; the entire manuscript was read by six steroid chemists outside the authors' laboratory ; individual chapters and sections were sent for comment to nearly every senior worker in each special branch of the field, and the authors generously acknowledge the very valuable help of these colleagues.

If I may offer one or two suggestions regarding further improvement of the book in its fifth edition, they are the following : the subject index, although very full, might be extended by the inclusion of an index of formulæ of the pattern used in Chemical Abstracts, and also an index of reactions; this might be of the type used in Theilheimer's "Synthetic Methods", or perhaps it would be more popular if it did not need a special technique for decoding. The authors have indexed some classes of reaction. including oxidation, reduction, hydrogenation, but not, for example, hydrolysis. Another useful addition to the index would be the names of 'elasses' of compounds, such as glucuronides and sulphates.

All steroid chemists will be still further indebted to Prof. and Mrs. Fieser for this wonderful achievemont, and it is hoped that many other chemists will take advantage of the mass of information and ideas which is laid before them in this book.

The minor criticisms of the index are rather like the comments of the traveller who directed attention to the ticking of the clock in a Rolls-Royce-for this is indeed a Rolls-Royce among monographs of organic chemistry.
W. KLyaf 